\title{
Correction to: Disinformation in Open Online Media
}

\author{
Max van Duijn (D), Mike Preuss (D), Viktoria Spaiser(D, \\ Frank Takes (D), and Suzan Verberne (D)
}

\section{Correction to: \\ M. van Duijn et al. (Eds.): Disinformation in Open Online Media, LNCS 12259, https://doi.org/10.1007/978-3-030-61841-4}

In the original online version of the chapter 5 was previously published non-open access. It was changed to open access retrospectively under a CC BY 4.0 license and, the presentation of Table 3 was different to that of Tables 2 and 4 . This has been corrected. In addition, Tables 5 - 8 have been moved from the main text to Appendix B, at the request of the authors.

The original version of the chapter 11 contained an error in Table 2, which also affected Section 3.1 and the Conclusion. The original figure in Table 2 indicated that one community was retweeting from a smaller number of accounts than the other communities. A recalculation following publication showed that the community was retweeting from a pool of about the same number of accounts as the other communities. This has been updated.

The updated version of these chapters can be found at https://doi.org/10.1007/978-3-030-61841-4_5

https://doi.org/10.1007/978-3-030-61841-4_11

(C) The Author(s) 2021

M. van Duijn et al. (Eds.): MISDOOM 2020, LNCS 12259, p. C1, 2021.

https://doi.org/10.1007/978-3-030-61841-4_19 